\title{
Avaliação do refluxo venoso superficial ao mapeamento dúplex em portadores de varizes primárias de membros inferiores: correlação com a gravidade clínica da classificação CEAP
}

\author{
Evaluation of venous reflux by color duplex scanning in patients with \\ varicose veins of the lower limbs: correlation with clinical severity by \\ CEAP classification
}

\section{Áurea Regina Teixeira de Andrade', Guilherme Benjamin Brandão Pitta ${ }^{2}$, Aldemar Araújo Castro ${ }^{3}$, Fausto Miranda Júnior ${ }^{4}$}

\section{Resumo}

Contexto: As alterações de pele manifestadas na insuficiência venosa crônica têm como etiologia mais comum o refluxo venoso. Alguns autores relatam que o refluxo venoso superficial é responsável por $40-60 \%$ das úlceras de perna nos portadores de varizes primárias.

Objetivo: Correlação do refluxo venoso superficial ao mapeamento dúplex com o quadro clínico (classificação CEAP - clínica, epidemiológica, anatômica e fisiopatológica) nos portadores de varizes primárias de membros inferiores.

Método: Estudo transversal e descritivo, desenvolvido em portadores de varizes primárias. As variáveis primárias foram: refluxo venoso e quadro clínico. O quadro clínico foi caracterizado por grupos: A, B e C, representados pelas categorias clínicas da CEAP. Dados complementares foram: tipos de refluxo em veias safenas magnas e parvas, conforme classificação de Engelhorn (2004). Testaram-se as hipóteses de inter-relações entre presença de refluxo e quadro clínico, utilizando-se os testes exato de Fisher e qui-quadrado no nível de $5 \%$ de probabilidade.

Resultados: Das 242 extremidades inferiores examinadas, 15 foram excluídas, totalizando 227 na amostra final. Noventa e nove $(83,9 \%)$ pacientes eram do sexo feminino. A média de idade foi de 50 anos, com mediana igual a 49 anos. Noventa e três extremidades (41\%) não apresentavam refluxo, e $134(59 \%)$ o apresentavam isolado e/ou associado. O refluxo isolado em veias perfurantes $(p=0,0008)$ e destas em associação com o refluxo em veias safenas magnas $(p<0,0001)$ estão significativamente relacionados à gravidade do quadro clínico.

Conclusão: Há correlação entre a presença do refluxo venoso superficial ao mapeamento dúplex e a gravidade do quadro clínico nos portadores de varizes primárias de membros inferiores.

Palavras-chave: Refluxo venoso, varizes primárias, mapeamento dúplex

\begin{abstract}
Background: Skin changes observable in chronic venous insufficiency have venous reflux as the most common etiology. Some authors have reported that reflux in the superficial venous system accounts for $40-60 \%$ of leg ulcers in patients with primary varicose veins.

Objective: To evaluate the correlation between superficial venous reflux and clinical status (CEAP classification - clinical, etiology, anatomy and pathophysiology) in patients with primary varicose veins of the lower limbs using duplex scanning.

Method: A cross-sectional and descriptive study was performed in patients with primary varicose veins. Primary variables were venous reflux and clinical status. Clinical status was characterized by groups $\mathrm{A}, \mathrm{B}$, and $\mathrm{C}$, represented by CEAP clinical categories. Types of venous reflux in the great and small saphenous veins were used as complementary data, according to Engelhorn's classification (2004). Hypotheses of interrelationship between incidence and types of reflux were statistically analyzed using Fisher's exact and chi-square tests. Significance was set at $0.05 \%$.

Results: Of 242 lower limbs, 15 were excluded, so that the final sample was comprised of 227 lower limbs. Ninety-nine (83.9\%) patients were female. Mean age was 50 years and median was 49 years. Reflux was absent in 93 limbs (41\%), and 134 (59\%) showed isolated and/or associated reflux. Isolated reflux in perforating veins $(p=0.0008)$ or in association with great saphenous vein reflux $(\mathrm{p}<0.0001)$ was significantly related to clinical status severity.

Conclusion: Duplex scan showed correlation between presence of superficial venous reflux and clinical status severity in patients with primary varicose veins of the lower limbs.
\end{abstract}

Keywords: Venous reflux, primary varicose veins, duplex scan.

1. Angiologista e cirurgiã vascular, Hospital das Clínicas Dr. José Augusto Leite, Aracaju, SE. Especialista, Angiologia e Cirurgia Vascular, SBACV e Associação Médica Brasileira (AMB).

2. Professor titular, Disciplina de Cirurgia Vascular, Universidade Federal de São Paulo - Escola Paulista de Medicina (UNIFESP-EPM), São Paulo, SP.

3. Doutor. Professor adjunto, Departamento de Cirurgia, Universidade Estadual de Ciências da Saúde de Alagoas Governador Lamenha Filho - Escola de Ciências Médicas de Alagoas (UNCISAL), Maceió, AL.

4. Mestre. Professor assistente, Disciplina de Metodologia da Pesquisa Científica, Departamento de Medicina Social, UNCISAL, Maceió, AL.

Não foram declarados conflitos de interesse associados à publicação deste artigo.

Artigo submetido em 04.01.08, aceito em 05.12.08.

J Vasc Bras. 2009;8(1):14-20.

Copyright (C 2009 by Sociedade Brasileira de Angiologia e de Cirurgia Vascular 


\section{Introdução}

O refluxo venoso desempenha papel crucial na magnitude dos sintomas e sinais da insuficiência venosa crônica dos membros inferiores, manifestando-se desde simples sensação de peso e cansaço até úlceras crônicas e extensas ${ }^{1}$.

As alterações de pele manifestadas na insuficiência venosa, decorrentes da hipertensão venosa, têm como etiologia mais comum o refluxo venoso ${ }^{2}$, quer no sistema superficial ${ }^{3}$, profundo $^{4}$ e em pérfurocomunicantes $^{5}$, isoladamente, ou em associação.

O refluxo venoso é preditivo para a gênese da úlcera venosa, principalmente se superior a $10 \mathrm{~mL} / \mathrm{s}$, independente se localizado em veias superficiais ou profundas ${ }^{6}$.

Com o mapeamento dúplex, alguns autores afirmam que o refluxo venoso superficial contribui com 40 a $60 \%$ das úlceras venosas nos pacientes portadores de varizes primárias de membros inferiores ${ }^{7-10}$.

Objetivou-se comparar o refluxo venoso superficial, avaliado pelo mapeamento dúplex, e o quadro clínico nos portadores de varizes primárias de membros inferiores, a fim de verificar se a presença e a extensão do refluxo estão relacionadas à gravidade clínica da classificação CEAP (clínica, epidemiológica, anatômica e fisiopatológica).

\section{Métodos}

Realizou-se um estudo transversal e descritivo, onde os pacientes foram atendidos em clínicas privadas. Incluímos, neste estudo, pacientes consecutivos portadores de varizes primárias com refluxo nas junções safenofemoral e/ou parvo-poplítea e/ou pérfuro-comunicantes e integridade do sistema venoso profundo através da ecografia vascular. Pacientes com insuficiência arterial e/ou linfática, gestantes, diabéticos, doentes psiquiátricos e com passado de qualquer intervenção cirúrgica prévia em membros inferiores foram excluídos.

Foram estudadas duas variáveis primárias: o refluxo venoso e o quadro clínico. Os dados complementares avaliados foram: tipos de refluxo em veias safenas magnas e parvas, conforme a classificação ultrassonográfica de Engelhorn ${ }^{11}$. O funcionamento valvular foi avaliado com o auxílio do mapeamento a cores do fluxo, pelas manobras de Valsalva e de compressão muscular manual distal ao posicionamento do transdutor, a fim de produzir e detectar refluxo no sistema venoso superficial ${ }^{11}$. O refluxo venoso foi definido como fluxo em sentido inverso com tempo superior a 0,5 segundos $^{12}$. O aparelho ultrassonográfico utilizado foi o SONOLINE Versa Pro Ultrasound Imaging System (Siemens Medical System), adaptado com sondas de 5, 7,5, 8 e $10 \mathrm{MHz}$. Utilizou-se ecógrafo com análise espectral Doppler, composto de unidade básica com transdutor linear de 7,5 e $10 \mathrm{MHz}$ (Diasonic Gateway) e sondas lineares de alta frequência $(7,5$ ou $10 \mathrm{MHz})$. Com o modo B (imagem bidimensional), estudou-se a anatomia venosa, e com o Doppler pulsado, o fluxo venoso, complementado pelo mapeamento a cores. Para avaliação do quadro clínico, foi utilizada a classificação clínica CEAP ${ }^{2,12}$, definida em:

- $\mathrm{C}_{0}$ quando não há sinais visíveis ou palpáveis de doença venosa;

- $C_{1}$ na presença de telangiectasias e veias reticulares;

- $\mathrm{C}_{2}$ na presença de veias varicosas;

- $\mathrm{C}_{3}$ na vigência de edema;

- $\mathrm{C}_{4}$ com alterações tróficas de pele;

- $\mathrm{C}_{5}$ com alterações tróficas de pele e úlcera cicatrizada;

- $\mathrm{C}_{6}$ com alterações tróficas de pele e úlcera ativa.

Foram pré-estabelecidos três grupos denominados:

- A, representado pelos pacientes da categoria $\mathrm{C}_{1}$ e $\mathrm{C}_{2}$ da classificação CEAP;

- $\mathrm{B}$, representado pelos pacientes da categoria $\mathrm{C}_{3}$ e $\mathrm{C}_{4}$ da classificação CEAP;

- C, representado pelos pacientes da categoria $\mathrm{C}_{5}$ e $\mathrm{C}_{6}$ da classificação CEAP.

O cálculo do tamanho da amostra foi estimado em 110 pacientes, baseados em trabalhos da literatura mundial ${ }^{1,7,9,13}$. Quanto à análise estatística, os dados gerais foram submetidos a uma análise descritiva e, posteriormente, foram testadas as hipóteses das inter-relações das variáveis: incidência e tipo de refluxo com o quadro clínico, utilizando-se o teste qui-quadrado, no nível de 5\% de probabilidade (alfa $=0,05 \%$ ), e o teste exato de Fisher, quando existissem células com valores inferiores a 5. 
Tabela 1 - Números e porcentagem de casos de ausência ou presença de refluxos isolados e associados em veias safenas (magnas e parvas) e perfurantes nos grupos A, B e C e valores obtidos pelo teste qui-quadrado em 227 membros inferiores

\begin{tabular}{|c|c|c|c|c|}
\hline \multirow[b]{2}{*}{ Ocorrência de refluxo } & \multicolumn{3}{|c|}{ Grupos, n (\%) } & \multirow[b]{2}{*}{ Total } \\
\hline & $\mathbf{A}$ & B & $\mathbf{C}$ & \\
\hline Ausência & $64(68,8)$ & $29(31,2)$ & $0(0,0)$ & $93(100)$ \\
\hline RVSMg & $9(52,9)$ & $7(41,2)$ & $1(5,9)$ & $17(100)$ \\
\hline RVSPv & $3(37,5)$ & $4(50,0)$ & $1(12,5)$ & $8(100)$ \\
\hline RVPf & $19(47,5)$ & $16(40,0)$ & $5(12,5)$ & $40(100)$ \\
\hline RVSMg + RVSPv & $2(25,0)$ & $5(62,5)$ & $1(12,5)$ & $8(100)$ \\
\hline RVSMg + RVPf & $7(17,5)$ & $22(55,0)$ & $11(27,5)$ & $40(100)$ \\
\hline RVSPv + RVPf & $0(0,0)$ & $3(75,0)$ & $1(25,0)$ & $4(100)$ \\
\hline $\mathrm{RVSMg}+\mathrm{RVSPv}+\mathrm{RVPf}$ & $2(11,8)$ & $13(76,4)$ & $2(11,8)$ & $17(100)$ \\
\hline Total & 106 & 99 & 22 & 227 \\
\hline
\end{tabular}

$\mathrm{RVSMg}=$ refluxo em veia safena magna; $\mathrm{RVSPv}=$ refluxo em veia safena parva; $\mathrm{RVPf}=$ refluxo em veias perfurantes.

\section{Resultados}

A amostra inicial foi constituída por 121 pacientes, totalizando 242 extremidades; todavia, três pacientes (seis extremidades) constituem o desvio da pesquisa, em virtude de o mapeamento dúplex não conter as informações necessárias para o preenchimento do formulário da coleta de dados. Nove extremidades foram excluídas devido a: refluxo em sistema venoso profundo $(n=2)$, trombose venosa profunda pregressa $(n=3)$ e intervenção cirúrgica prévia nos membros inferiores $(n=4)$.

$\mathrm{Na}$ análise descritiva dos dados, verificou-se que 99 pacientes eram do sexo feminino (83,90\%), e 19 , do sexo masculino (16,19\%). A idade média foi de 50 anos, com $50 \%$ dos pacientes situados na faixa dos 40-59 anos, e a mediana, igual a 49 anos. Nossa amostra final foi constituída por 227 membros inferiores, cujo refluxo venoso superficial esteve ausente em 93 (41\%) e presente em 134 (59\%), isoladamente, ou em associação, assim distribuídos: 17 (7,5\%), 8 (3,5\%) e $40(17,6 \%)$ apresentavam, respectivamente, refluxo em veias safenas magnas, veias safenas parvas e veias perfurantes, de forma isolada.

Oito extremidades inferiores (3,5\%) apresentavam refluxo em ambas as safenas, magna e parva; $40(17,6 \%)$, em veias safenas magnas e perfurantes; quatro $(1,7 \%)$ apresentavam associações de refluxo em veias safenas parvas e perfurantes; e em 17 (7,5\%), foram diagnosticadas associações dos três tipos de refluxo, de acordo com a Tabela 1.

A Tabela 1 demonstra todos os refluxos distribuídos nas respectivas classes clínicas. Aplicamos o teste estatístico qui-quadrado, que foi estatisticamente significante para todos os refluxos superficiais. Contudo, desdobramos a Tabela 1 e aplicamos, para cada tipo de refluxo, separadamente, o teste qui-quadrado e o teste exato de Fisher. Constatamos que os refluxos estatisticamente significantes foram a associação dos refluxos de veias safenas magnas e perfurantes e o de veias perfurantes, isoladamente.

A tabela do qui-quadrado foi desdobrada, e novos testes foram realizados, a fim de evidenciar quais das associações de refluxo estão mais relacionadas à gravidade do quadro clínico. Observamos que as ocorrências da associação de refluxo entre as veias safenas magnas e perfurantes (qui-quadrado $=43,5 ; \mathrm{p}=0,001$ ) e a ocorrência de refluxo em veias perfurantes, isoladamente (qui-quadrado $=14,3 ; \mathrm{p}<0,0008$ ), estão significativamente relacionadas à gravidade do quadro clínico, como demonstrado nas Tabelas 2 e 3, respectivamente.

Quanto à ocorrência de refluxo em veias perfurantes nas categorias clínicas e a sua localização nos membros 
Tabela 2 - Número e porcentagens de casos (ausência ou presença) de refluxo associado em veias safenas magnas e perfurantes dentro dos grupos A, B e C e valores obtidos pelo teste qui-quadrado (qui-quadrado = 43,5; p < 0,0001) em 133 dos membros inferiores

\begin{tabular}{lcccc}
\hline & \multicolumn{3}{c}{ Grupos, $\mathbf{n}(\%)$} & \\
\cline { 2 - 4 } $\begin{array}{l}\text { Ocorrência de refluxo em } \\
\text { veias safenas magnas e } \\
\text { perfurantes }\end{array}$ & A & B & C & Total \\
\hline Ausência de refluxo & $64(90,1)$ & $29(56,9)$ & $0(0,0)$ & 93 \\
Presença de refluxo & $7(9,9)$ & $22(43,1)$ & $11(100)$ & 40 \\
Total & $71(100)$ & $51(100)$ & $11(100)$ & 133 \\
\hline
\end{tabular}

Qui-quadrado $=43,5 ; \mathrm{p}<0,0001$.

Tabela 3 - Número e porcentagem de casos de refluxos (ausentes ou isolados) de veias perfurantes nos grupos A, B e C e valores obtidos pelo teste qui-quadrado em 133 membros inferiores

\begin{tabular}{|c|c|c|c|c|}
\hline \multirow{2}{*}{$\begin{array}{l}\text { Ocorrência de refluxo em } \\
\text { veias perfurantes }\end{array}$} & \multicolumn{3}{|c|}{ Grupos, n (\%) } & \multirow[b]{2}{*}{ Tota } \\
\hline & A & B & C & \\
\hline Ausência de refluxo & $64(77,1)$ & $29(64,4)$ & $0(0,0)$ & 93 \\
\hline Refluxo isolado & $19(22,9)$ & $16(35,6)$ & $5(100)$ & 40 \\
\hline Total & $83(100)$ & $45(100)$ & $5(100)$ & 133 \\
\hline
\end{tabular}

Qui-quadrado $=14,3 ; \mathrm{p}=0,0008$.

examinados, observamos uma predominância nas categorias $\mathrm{C}_{2}, \mathrm{C}_{3}$ e $\mathrm{C}_{4}$, com localização na face medial em todas as classes clínicas, conforme demonstrado na Figura 1.

Em dados complementares, foram avaliados os tipos de refluxos em veias safenas magnas e parvas, de acordo com Engelhorn. Os 82 casos de refluxos em veias safenas magnas foram classificados e distribuídos, conforme a Figura 2. Observou-se maior frequência do refluxo tipo II (proximal), com $31,7 \%$ de ocorrência, seguida do tipo IV (segmentar), em $28,1 \%$, e do tipo VI (difuso), em $25,6 \%$ dos casos.

O refluxo em veias safenas parvas também foi classificado quanto ao tipo (Figura 3), verificando-se maior frequência do tipo II (proximal), com 37,8\% dos casos. As ocorrências de refluxo do tipo I (veia de Giacomini) e do tipo IV foram de 18,9 e $16,2 \%$, respectivamente. Não houve registro de nenhum caso de refluxo do tipo

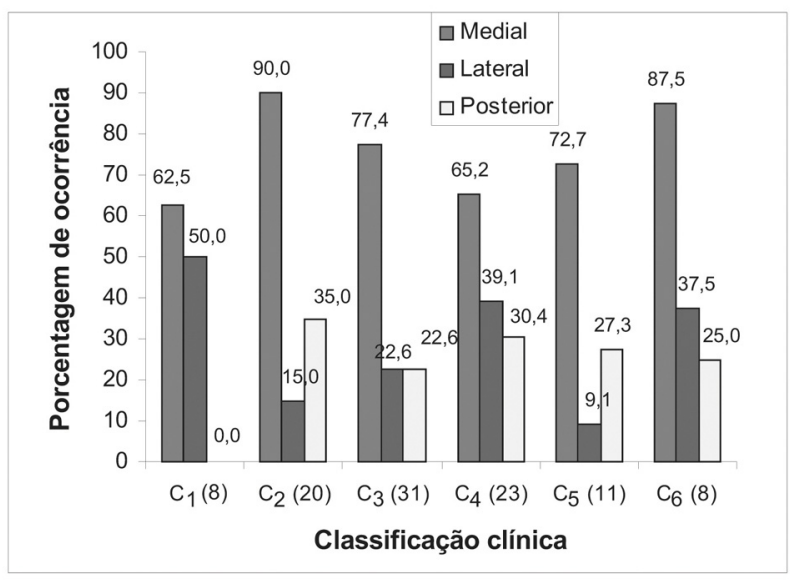

Figura 1 - Percentuais de refluxo em veias perfurantes de acordo com a localização nos membros inferiores e a classificação clínica (CEAP)

V (multissegmentar). Os refluxos tipo III (distal) e VI (difuso) ocorreram em 13,3\% da amostra. 


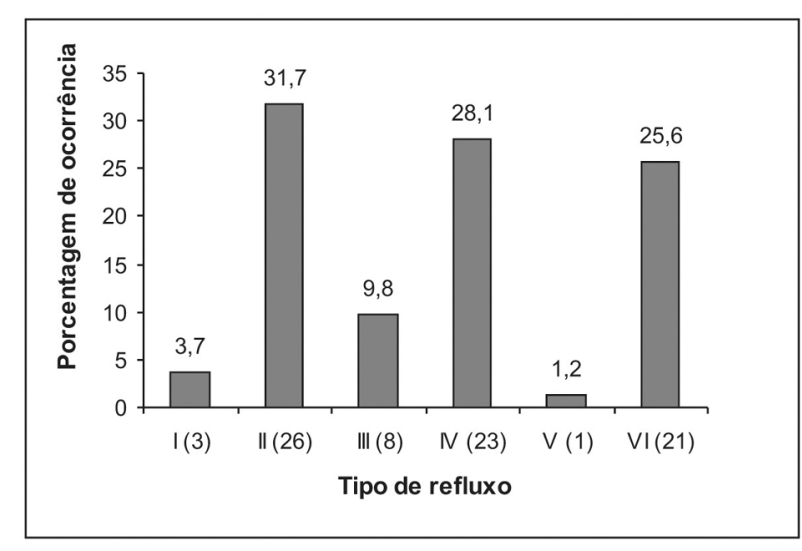

$\mathrm{I}$ = perijunção; $\mathrm{II}$ = proximal; $\mathrm{III}$ = distal; $\mathrm{IV}$ = segmentar, $\mathrm{V}$ = multissegmen$\operatorname{tar} ; \mathrm{VI}=$ difuso.

Figura 2 - Porcentagens dos tipos de refluxo na veia safena magna em 82 membros inferiores

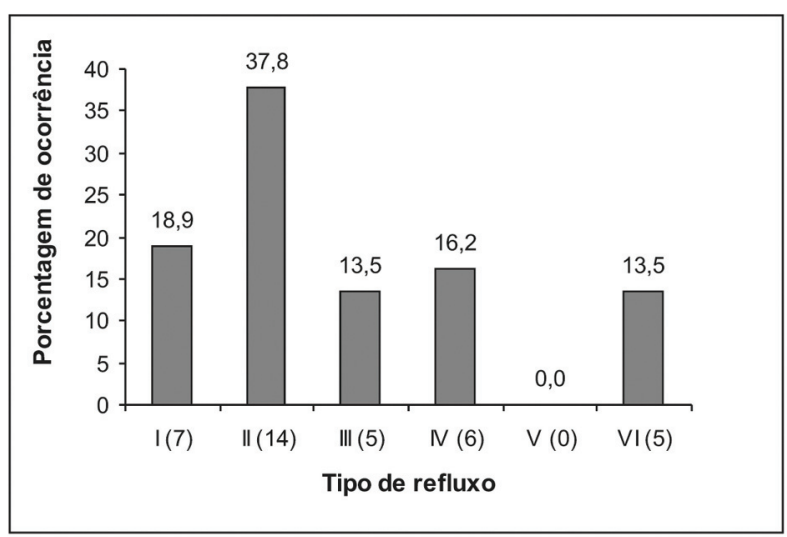

$\mathrm{I}$ = veia de Giacomini; $\mathrm{II}$ = proximal; $\mathrm{III}$ = distal; IV = segmentar; $\mathrm{V}$ = multissegmentar; VI = difuso.

Figura 3 - Porcentagens de refluxo na veia safena parva em 37 membros inferiores

\section{Discussão}

O exame clínico vascular foi realizado dentro dos moldes semiológicos clássicos, e o quadro clínico, estratificado através da classificação CEAP, categoria clínica, porque é mundialmente conhecida, mais completa e permite estudos interinstitucionais ${ }^{2,14,15}$.

O mapeamento dúplex foi o método de escolha na investigação da doença venosa, por ser sensível, específico e fornecer dados anatomofuncionais dos sistemas venosos ${ }^{2}$. Os pacientes foram examinados nas posições supina e ortostática, segundo Sarquis ${ }^{16}$.
O refluxo venoso superficial é considerado atualmente a causa mais frequente das alterações morfofuncionais, sendo responsável por cerca de 40 a $60 \%$ das lesões tróficas perimaleolares dos portadores de varizes primárias de membros inferiores ${ }^{7-10}$. Evidenciamos relação significativa entre a gravidade do quadro clínico e a presença de refluxo venoso superficial na totalidade da amostra (qui-quadrado $=58,8 ; \mathrm{p}<0,0001$ ). Ainda responsabilizando o sistema venoso superficial pelas alterações de pele, Labropoulos et al. ${ }^{17}$ avaliaram pacientes com refluxo venoso e trombose venosa prévia, relacionando-os com a úlcera venosa. Observaram uma baixa incidência de úlcera nos pacientes com ausência de refluxo venoso superficial, apesar da obstrução do sistema venoso profundo.

Em nosso estudo, a ocorrência de refluxo em veias safenas magnas, isoladamente, ou em associação com a insuficiência de veias safenas parvas não foi estatisticamente significante; todavia, Labropoulos et al. ${ }^{18}$ observaram que, numa amostra de 255 membros inferiores com insuficiência venosa superficial, a úlcera venosa esteve presente quando a veia safena magna apresentava refluxo em toda a sua extensão ( $8 \%$ ), e essa frequência aumentou para $14 \%$ quando da associação de ambos os refluxos em veias safenas magnas e parvas. A associação de refluxo em veias safenas magnas e perfurantes, na nossa observação, foi estatisticamente significante (qui-quadrado $=43,5 ; \mathrm{p}<0,0001$ ) e determinou a gravidade da doença venosa. Os nossos dados corroboram as observações de Hanrahan et al. ${ }^{10}$, quando analisaram 95 membros inferiores com úlcera venosa e, destes, 19\% tinham associação de refluxo em veias safenas magnas e perfurantes.

Em relação ao refluxo de veias perfurantes, Stuart et al. ${ }^{19}$, avaliando um grupo de pacientes pertencentes às categorias $\mathrm{C}_{0}$ a $\mathrm{C}_{6}$ da classificação CEAP, observaram que a gravidade da doença estava relacionada com o número de perfurantes insuficientes por membro e com a associação de veias superficiais, principalmente com a insuficiência de veia safena parva. Nossas observações estão em concordância com os trabalhos de Lees \& Lambert ${ }^{9}$ e Hanrahan et al. ${ }^{10}$ no que se refere à ocorrência do refluxo em perfurantes localizadas em face medial 
dos membros inferiores nos portadores de varizes primárias.

Quanto à localização das veias perfurantes insuficientes, Delis et al. ${ }^{20,21}$, Sandri et al. ${ }^{22}$ e Stuart et al. ${ }^{19}$ observaram que a face medial dos membros inferiores foi a mais frequente. Entretanto, no nosso estudo, essa localização não definiu a gravidade da doença. Observa-se refluxo em veias perfurantes, isolado ou em associação em todas as categorias clínicas, desde $\mathrm{C}_{1}$ a $\mathrm{C}_{6}$ da classificação CEAP; no entanto, a ausência de refluxo nessas veias, isoladamente, foi predominante na categoria $\mathrm{C}_{1}$ da CEAP. Labropoulos et al. ${ }^{23}$ relataram que a presença de veias perfurantes insuficientes é rara em indivíduos livres de sinais e/ou sintomas da doença venosa. Enfatizando, Stuart et al. ${ }^{19}$ demonstraram que pacientes portadores de telangiectasias apresentavam apenas $5 \%$ de perfurantes insuficientes, enquanto os que apresentavam úlcera cicatrizada ou aberta contribuíram com $77 \%$ da insuficiência das perfurantes da amostra. Contudo, ainda permanece controverso o papel das perfurantes insuficientes na gravidade da doença venosa crônica dos membros inferiores. Vale ressaltar que a falta de identificação do refluxo em veias perfurantes no préoperatório de varizes de membros inferiores contribui para sua recorrência, sendo imperativa a avaliação destas no leito venoso, segundo Hanrahan et al. ${ }^{24}$.

Observamos que o refluxo em veia safena parva não foi significativo para determinar a gravidade do quadro clínico, quando isolado ou em associação com as veias safenas magnas e perfurantes; no entanto, Bass et al. ${ }^{25}$, avaliando 20 membros inferiores com úlcera venosa, em maléolo lateral, observaram que todos os membros apresentavam refluxo isolado em veia safena parva. Em nosso estudo, a presença de refluxo isolado em veia safena parva foi de 3,5 e de $12,8 \%$ em associação com outros refluxos. Os testes estatísticos qui-quadrado e exato de Fisher não comprovam a relação entre presença de refluxo em veia safena parva e gravidade do quadro clínico.

Avaliando-se os modelos de refluxo em veias safenas, encontramos o tipo II (proximal) como o mais frequente para ambas as safenas, corroborando os achados de Engelhorn. Sabendo-se que o refluxo venoso superficial tem responsabilidade clínica e hemodinâmica significativa na gênese das lesões de pele e que alguns tipos de refluxo são preditores para a formação da úlcera venosa, poderemos intervir em tempo hábil com medidas preventivas ou terapêuticas, a fim de melhorar a grande morbidade que a insuficiência venosa crônica impõe aos seus portadores.

Estamos distantes ainda de estabelecer padrões de correlações entre o exame clínico e o mapeamento dúplex, principalmente em virtude do polimorfismo da insuficiência venosa crônica, por isso justifica-se o incentivo a novas pesquisas nesse aspecto, a fim de desenvolver estudos que estabeleçam o risco relativo de determinado grupo observacional desenvolver a úlcera venosa de membros inferiores. Desse modo, estaríamos desenvolvendo uma medicina preventiva num futuro bem próximo.

\section{Conclusões}

Há correlação entre a presença do refluxo venoso superficial ao mapeamento dúplex e a gravidade do quadro clínico nos portadores de varizes primárias de membros inferiores.

Há predominância do refluxo em veias perfurantes, isoladamente, e em associação com o refluxo em veias safenas, ambos relacionados diretamente à gravidade da doença venosa crônica dos membros inferiores.

\section{Referências}

1. Labropoulos N, Giannoukas AD, Delis K, et al. Where does venous reflux start? J Vasc Surg. 1997;26:736-42.

2. Porter JM, Moneta GL. Reporting standards in venous disease: an update. International Consensus Committee on Chronic Venous Disease. J Vasc Surg. 1995;21:635-45.

3. Sethia K, Darke SG. Long saphenous incompetence as a cause of venous ulceration. Br J Surg. 1984;71:754-5.

4. Negus D, Friedgood A. The effective management of venous ulceration. Br J Surg. 1983;70:623-7.

5. Cockett FB. The pathology and treatment of venous ulcers of the leg. Br J Surg. 1955;43:260-78.

6. Vasdekis SN, Clarke GH, Nicolaides AN. Quantification of venous reflux by means of duplex scanning. J Vasc Surg. 1989;10:670-7.

7. Shami SK, Sarin S, Cheatle TR, Scurr JH, Smith PD. Venous ulcers and the superficial venous system. J Vasc Surg. 1993; 17:487-90. 
8. Wong JK, Duncan JL, Nichols DM. Whole-leg duplex mapping for varicose veins: observations on patterns of reflux in recurrent and primary legs, with clinical correlation. Eur J Vasc Endovasc Surg. 2003;25:267-75.

9. Lees TA, Lambert D. Patterns of reflux in limbs with skin changes associated with chronic venous insufficiency. Br J Surg. 1993;80:725-8.

10. Hanrahan LM, Araki CT, Rodriguez AA, Kechejian GJ, LaMorte WW, Menzoian JO. Distribution of valvular incompetence in patients with venous stasis ulceration. J Vasc Surg. 1991;13:805-11; discussion 811-2.

11. Engelhorn CA, Engelhorn AL, Cassou MF, Zanoni CC, Gosalan CJ, Ribas E. Classificação anatomofuncional da insuficiência das veias safenas baseada no eco-Doppler colorido, dirigida para o planejamento da cirurgia de varizes. J Vasc Bras. 2004;3:13-9.

12. Labropoulos N, Tiongson J, Pryor L, et al. Definition of venous reflux in lower-extremity veins. J Vasc Surg. 2003;38:793-8.

13. Labropoulos N, Kang SS, Mansour MA, Giannoukas AD, Buckman J, Baker WH. Primary superficial vein reflux with competent saphenous trunk. Eur J Vasc Endovasc Surg. 1999;18:201-6.

14. Rutherford RB, Padberg FT Jr, Comerota AJ, Kistner RL, Meissner MH, Moneta GL. Venous severity scoring: An adjunct to venous outcomes assessment. J Vasc Surg. 2000;31:1307-12.

15. Eklöf B, Rutherford RB, Bergan JJ, et al. Revision of the CEAP classification for chronic venous disorders: consensus statement. J Vasc Surg. 2004;40:1248-52.

16. Sarquis AL. Avaliação pré e pós-operatória no tratamento cirúrgico conservador de varizes tronculares com o duplex scan a cores. Cir Vasc Angiol. 1996;12:9-11.

17. Labropoulos N, Leon M, Nicolaides AN, et al. Venous reflux in patients with previous deep venous thrombosis: correlation with ulceration and other symptoms. J Vas Surg. 1994;20:20-6.
18. Labropoulos N, Leon M, Nicolaides AN, Giannoukas AD, Volteas N, Chan P. Superficial venous insufficiency: correlation of anatomic extent of reflux with clinical symptoms and signs. J Vasc Surg. 1994;20:953-8.

19. Stuart WP, Adam DJ, Allan PL, Ruckley CV, Bradbury AW. The relationship between the number, competence, and diameter of medial calf perforating veins and the clinical status in healthy subjects and patients with lower-limb venous disease. J Vas Surg. 2000;32:138-43.

20. Delis KT, Ibegbuna V, Nicolaides AN, Lauro A, Hafez H. Prevalence and distribution of incompetent perforating veins in chronic venous insufficiency. J Vasc Surg. 1998;28:815-25.

21. Delis KT. Perforator vein incompetence in chronic venous disease: A multivariate regression analysis model. J Vasc Surg. 2004;40:626-33.

22. Sandri JL, Barros FS, Pontes S, Jacques C, Salles-Cunha SX. Diameter-reflux relationship in perforating veins of patients with varicose veins. J Vasc Surg. 1999;30:867-74.

23. Labropoulos N, Delis KT, Nicolaides AN. Venous reflux in symptom-free vascular surgeons. J Vasc Surg. 1995;22:150-4.

24. Hanrahan LM, Araki CT, Fisher JB, et al. Evaluation of the perforating veins of the extremity using high resolution duplex imaging. J Cardiovasc Surg (Torino). 1991;32:87-97.

25. Bass A, Chayen D, Weinmann EE, Ziss M. Lateral venous ulcer and short saphenous vein insufficiency. J Vasc Surg. 1997;25:654-7.

Correspondência:

Áurea Regina Teixeira de Andrade

Rua Dr. Wilson Rocha, 950/1001, Bairro Grageru

CEP 49025130 - Aracaju, SE

E-mail: aureareginata@yahoo.com.br 